\title{
Allocate Fair Payoff for Cooperation in Wireless Ad Hoc Networks Using Shapley Value
}

\author{
Jianfeng Cai \\ Department of Computer Science \\ Texas A\&M University \\ College Station, TX, 77843-3112 \\ j0c1194@cs.tamu.edu
}

\author{
Udo Pooch \\ Department of Computer Science \\ Texas A\&M University \\ College Station, TX, 77843-3112 \\ pooch@cs.tamu.edu
}

\begin{abstract}
In wireless mobile ad hoc networks (MANET), energy is a scarce resource. Though cooperation is the basis of network services, due to the limited energy reserve of each node, there is no guarantee any given protocols would be followed by nodes managed by different authorities.

Instead of treating the selfish nodes as a security concern and trying to eliminate them, we propose a novel way to encourage cooperative works - rewarding service providers according to their contributions. Nodes in a MANET can form coalitions to reduce aggregate transmission power on each hop along a route. The payment of each node in a coalition is determined by using Shapley Value, a well-known concept in game theory for allocating payoff for each member in a cooperative coalition.
\end{abstract}

We present the Contribution rewArd routing Protocol with Shapley Value (CAP-SV) in this paper. It achieves the objective of truthfulness. The performance of CAP-SV is studied by simulations using ns-2. Analysis and experimental results show a routing protocol with the consideration of the incentives of individual nodes stimulates cooperation and improves network lifetime without significantly diminishing the performance of the whole network.

\section{Keywords}

ad hoc networks, selfish nodes, energy, Shapley value, game theory

\section{INTRODUCTION}

In mobile ad hoc networks (MANET), network services are fulfilled by the cooperation of all nodes instead of pre-deployed facilities. Due to the limited radio transmission range, data packets are usually forwarded by multiple intermediate nodes before they reach the destination. Packet transmission does not come for free. In addition to the bandwidth and computational cost, energy is spent by each forwarding node.

Energy is a precious resource in MANET environment because the amount is limited and it cannot be replenished in a short time. Researchers $[18,22]$ show that the communication component is the main source of energy consumption. Even when a node is in idle state, neither sending nor receiving any packets, the energy consumption is still significant. Only when a node shuts down its radio, can the power level drop dramatically.
Some routing protocols $[4,5,7,8]$ address the energy conservation problem by saving energy globally. An individual must follow some pre-defined procedures though it may cost more energy from a local point of view. This is not always the case in general ad hoc setting. For example, in a technical conference, an ad hoc network is established by some notebooks which belong to different people. If a node generates a huge volume of network traffic, it may deplete the energy of other intermediate nodes. The owner of a relay node would not be happy if his/her notebook runs out of battery for processing others' business. He/she may change the configuration of the notebook to ban the participation of routing and forwarding services and only spend energy on transferring or receiving his/her own data.

Selfish nodes are common within the ad hoc context because they are managed by different authorities. Each of them wants to maximize its profit, which is the payment minus its cost incurred by serving a network. Therefore, the networkwide services should not come for free. In this paper we argue that the work of a forwarding node should bring back some payoff to it. In another word, if a node uses its own energy for others, it should be compensated. The earning would enhance the incentive of helping others. We stimulate nodes to provide services for others by giving payment to the providers based on their contributions. If a selfish node feels that following a mechanism will bring back not less than what it can get by playing alone, it will have no intention to break the rules.

Selfish nodes are different from malicious nodes, though both of them may impair the performance of a network. Selfish nodes are rational because they do not attempt to attack others on their own cost. Malicious nodes always try to degrade network services even with huge cost.

We take the game-theoretical approach to design a truthful mechanism for our routing protocol. Truthfulness means that revealing true information is the best interest of the nodes in a MANET. In game theory, each game gives rules and payoff functions. A utility of a player corresponds to the received payment minus the incurred cost. A player may try to cheat in a game to maximize its utility. However in a truthful mechanism, cheating cannot bring more profits to a player than telling the truth. Consequently, a node would lose the intension to lie and only play correctly. 
We argue that selfish nodes intend to accept payments for forwarding packets for others to cover the cost incurred by the data transmission. We show a way to form cooperative coalitions and to divide the payment of a coalition fairly to each member.

We use the Shapley Value, which is the solution of cooperative games, to allocate the payoff for each network service provider. The individual payoff is determined according to how much a node brings to the coalition by its participation. Due to the characteristics of wireless propagation, multi-hop transmission may save the total transmission power. We allow an intermediate node to redirect the route if this redirection can save aggregate transmission power. We measure the transmission power reduction and use it as a gauge of the payoff. The more power saved by the redirection, the more payoff a node earns. The redirecting nodes on the same hop form a coalition which has properties fallen into the scope of what the Shapley Value addresses. Then we use the Shapley Value to decide the amount of payoff for each node in the redirection coalition. This is the motivation of this research.

In our scheme, a node has incentives to earn payoff from its works, such as forwarding packets for other nodes, and try to beat others by making more fortune. The form of wealth could be some virtual currency (e.g. Nuglets [9]). Each node knows the wealth of itself and its neighbors. If it is the richest or among top richest nodes within neighborhood, it may take a break and go to sleep while others continue to work to keep up with it. The richest ones can relax until they become less rich compared to their neighbors. Afterwards, they come back to work again. Based on all these considerations, we propose the Contribution rewArd routing Protocol with Shapley Value (CAP-SV) for wireless ad hoc networks. We implement CAP-SV in ns-2 network simulator [2] and evaluate its performance with AODV[26] as a reference.

In this paper, we present a way for each node to accumulate wealth. However, how to redeem the fortune a node earns is not addressed. We believe the process for a node to spend its wealth will bring benefit for network service management. For example, rich nodes can obtain better quality of services in a network than poor nodes. To be a rich one gives all nodes a stimulus to work for the whole network. We do not tackle this issue at this point because we believe it is worthy of further research works. And by taking it cautiously, it will not influence the energy saving we achieve in this paper.

The remainder of this paper is organized as follows: Section 2 reviews related works. A brief introduction to the Shapley Value is given in section 3 . Section 4 presents CAP-SV protocol design. The truthfulness of CAP-SV is discussed in section 5. Performance evaluation is shown in section 6 . Section 7 summarizes and points out some future works.

\section{RELATED WORK}

PARO [5] reduces the aggregate transmission power on each hop by taking advantage of multi-hop transmission. It allows a route to be redirected by intermediate nodes. The nodes in PARO must always turn on their radios, so the idle energy consumption would be significant. Since the nodes in ad hoc networks have intention to be selfish, they may not volunteer to redirect a path all the time. SPAN [4] elects coordinators to form a forwarding backbone. Noncoordinators can enter the doze state to save energy. SPAN does not use adaptive power, thus every node keeps a uniform power level as long as it is on. This may cost more energy than necessary and cause more radio interference. In [3], the cone-base distributed topology control algorithm reduces the degree of all the nodes in a wireless ad hoc network in order to reduce the interference and save energy. GAF [7] divides an area into virtual grids. Nodes in the same grid are the equivalent routers and shift in three states: active, sleeping and discovering to forward network traffic in turn. A power saving MAC layer protocol NPSM is presented in [6] to remove ATIM window from IEEE 802.11 PSM in order to improve network throughput and increase the energy efficiency. [8] studies the effects of power-aware metrics on MANET routing.

All the algorithms and protocols above assume nodes always follow some pre-defined procedures without consideration of nodes' intention of saving energy for their own usage. [19] calls this type of protocols compulsory protocols. In real world a protocol may not have the authority to force all nodes to do what they are supposed to do. In [13], researchers show that even a small portion of mis-behaving nodes can degrade the network performance dramatically. $[24,25]$ attack the security routing problem by establishing countermeasure mechanisms against malicious nodes. However, selfish nodes are different from malicious nodes because they are rational.

Some economic concepts have already been introduced into this research area. In $[9,10,19]$, the cooperation between nodes is no longer taken for granted. Instead, mechanisms are designed to stimulate the cooperative works. In [9], network services are traded on each hop toward the destinations.

Game theory $[15,17]$ gives us methods to model cooperative and non-cooperative games between different parties. Some interesting algorithmic issues of mechanism design are discussed in [30]. [19] introduces game theory to design an incentive-compatible scheme for resource management in MANET. Ad hoc-VCG [29] adopts game-theoretic setting and achieves cost-efficiency and truthfulness in MANET routing. Distributed mechanism design and applications are presented and analyzed in [31, 32, 33].

\section{SHAPLEY VALUE}

In Lloyd Shapley's 1953 paper, he proposed a cooperative solution concept, Shapley Value, which assigns a unique result to each finite n-player cooperative game. The outcome is payoff vectors for each node, which can be regarded as expected gains from the game and correspond to the contribution of each player to the game. In his paper, Shapley also proved that the Shapley Value is the only one to satisfy all the three axioms of cooperative games: efficiency, symmetry, additivity [16]. The function of the Shapley Value is shown below.

$$
\phi_{i}(v)=\sum_{i \in S} \frac{(|S|-1) !(n-|S|) !}{n !}[v(S)-v(S-\{i\})]
$$


In the function, $|S|$ is the size of a coalition or the number of members in a coalition. $\mathrm{v}(\mathrm{S})$ is the minimum worth which the coalition $\mathrm{S}$ can guarantee its members, and $\mathrm{v}(\mathrm{S}-\mathrm{i})$ is the payoff secured by a coalition with the same members in $\mathrm{S}$ except i.

In the Shapley Value, all the coalitions are regarded equal which means they have the same probability to appear. The first part of the formula can be interpreted as the probability of a coalition containing player i with the size of $|S|$. The second part is the payoff difference between the coalitions with and without player $i$, which measures the contribution from i to the coalition. The bigger the difference, the more the player contributes to its coalition, then the more payoff it earns. The interpretation details of the Shapley Value can be found in $[15,16,17]$.

Binding the contribution of a player with a fair value stimulates the incentive of it to cooperate with others. Since the Shapley Value turns out to do rather well on the payoff allocation, we apply it to determine how much each node can earn for cooperating with others in a MANET.

\section{CAP-SV PROTOCOL DESIGN}

We assume the links between different nodes are bi-directional. Modern radio technology allows nodes in a MANET to vary their transmission power levels. Each receiver can measure the received signal strength though it may not know the coming direction of that signal. If the receiver additionally knows the transmission power level, it can estimate the minimal power required for a message between these two nodes. Thus, in CAP-SV, each sender adds the emission power information to the header of the packet; a receiver assesses the received signal strength, calculates the minimal required power and informs the sender this value.

In CAP-SV, every node broadcast hello messages to all neighbors periodically. The wealth information is included in a hello message. The payment of each forwarding node only delivered after a packet is received by the intended destination. We assume some virtual currency systems would take care of this issue. All nodes have uniform radio components. Thus, they would consume the same energy for sending a data unit. As a result, maximizing the payment earned by a node would maximize its profit.

\subsection{Power Saving in Radio Propagation}

Due to the characteristics of radio channel propagation models, transferring a packet may cost less power if it is relayed by multiple intermediate nodes other than transferred on a single hop. In the two-ray ground reflection model, the power of received signal $\operatorname{Pr}$ is expressed as the formula below $[28]$.

$$
P_{r}=\frac{P_{t} \times G_{t} \times G_{r} \times\left(H_{t}^{2} \times H_{r}^{2}\right)}{d^{4}}
$$

$\mathrm{d}$ is the distance from the transmitter to the receiver; $P_{t}$ is the transmission power of the transmitter. $G_{t}$ and $G_{r}$ are transmitter and receiver's antenna gains. $H_{t}$ and $H_{r}$ are the height of the antennas.
The received power is inversely proportional to the $d^{4}$. If the distance decreases by a half then the transmission power can reduce to be the $\frac{1}{16}$ of the original value while keeping the same $P_{r}$. Reducing transmission power can reduce the radio interference and increase the network lifetime. Although some researchers $[3,5,12]$ already notice dynamic power level is a promising property toward an energy-efficient MANET, we take a step further to introduce the Shaley Value to enrich theories in this field.

If we use $K$ to represent the constant factors in the function (2), we have:

$$
P_{r}=\frac{P_{t} \times K}{d^{4}}
$$

Moreover, if the threshold of received signal strength, $P_{r t h d}$, is known the minimal transmission power, $P_{t m i n}$, used by sender would be:

$$
P_{t m i n}=\frac{P_{r t h d} \times P_{t}}{P_{r e c}}
$$

A node is able to compute the minimal emission power used to reach another node as long as the ongoing emission power and received power are known.

\subsection{Routing Procedures}

CAP-SV is a reactive routing protocol in which only when a source node has packets to send, is a route discovery process initiated. CAP-SV consists of three phases, route discovery, coalition establishment and role rotation.

\subsubsection{Route discovery}

In CAP-SV, initially, a source node that has packets to send broadcast a route request, RREQ, into the network. The transmission power used by the sender is included in the RREQ. Each node receiving the RREQ measures the received signal power and checks whether it is the destination. If it is not, it would forward the request by broadcasting it again. The transmission power level would be updated as that used by forwarding nodes. When the destination node receives the RREQ, a route reply message, RREP, is created and sent out by unicast. The RREP would be forwarded on a reverse route to the source node. Each node along the route records the destination node and next hop toward it. After a route is established, the source node can send its packets on this route.

The process described above is similar to any other distance vector protocols, such as AODV [26]. However, there are two points distinguishing CAP-SV from other protocols. A sender of a packet always attaches its transmission power level information with the packet and each receiver would measure the received signal power level to calculate the minimal power to reach the sender. Secondly, we allow the intermediate nodes to redirect the route on each hop and form cooperative coalitions on the fly. The payoff of each node in a coalition is calculated by applying the Shapley Value. Those nodes picked by the initial route discovery process are also paid a certain amount of payments, which are enough to cover their cost. This guarantees normal routing nodes would play truthfully. 


\subsubsection{Coalition establishment}

\section{A. Overhearing and redirection}

In a wireless ad hoc network, a node can overhear the network traffic within its radio range though the packets may not be sent to it. An intermediate node, using CAP-SV, overhears the route reply messages and checks whether a route through itself would offer a power saving path compared to the old one. We are interested in the total transmission power for one hop.

Let $N_{s}$ be the sender node of a hop and on the other end is node $N_{r}$. Node $N_{i}$ is an intermediate node between $N_{s}$ and $N_{r}$. The minimum power for packet transmission from $N_{s}$ to $N_{r}$ is $P_{s r}$; the minimum power for sending packets from $N_{s}$ to $N_{i}$ is $P_{s i}$, and $P_{i r}$ is the minimum power for packet transmission from $N_{i}$ to $N_{r}$. Then if the path is redirected by $N_{i}$, the following condition must be satisfied.

$$
P_{s i}+P_{i r} \leq \alpha \times P_{s r}
$$

$\alpha$ is a constant threshold that has a value within $[0,1]$. The intermediate node, $N_{i}$, sends a redirection message to both $N_{s}$ and $N_{d}$ to inform them a more power-efficient route and indicate, in the message, the minimum power which could be used by them to communicate with it. Upon receiving the redirection packets, the end nodes update their routing tables and make the route go through $N_{i}$.

$\alpha$ has impact on the performance of the protocol. If $\alpha$ is too small and the node density is high, lots of intermediate nodes try to redirect the route and send out redirection packets though the improvement may be slight. This would cause lots of packet collisions and decrease the network throughput.

For each redirection attempt, we define an improve ratio $R_{\text {improve }}$ as the ratio of the reduced transmission power against the minimum transmission power over the original hop. $R_{\text {improve }}$ is included in redirection packets. In the example with the three nodes, $N_{s}, N_{d}$ and $N_{i}$,

$$
R_{\text {improve }}=\frac{P_{s d}-\left(P_{s i}+P_{i d}\right)}{P_{s d}}=1-\frac{P_{s i}+P_{i d}}{P_{s d}}
$$

To further reduce packet collisions, we let each redirecting node delay for a while before the transmission of redirection packets. The delay is corresponding to $R_{\text {improve }}$ of that round. The greater the $R_{\text {improve }}$, the shorter the delay. The path redirection process works iteratively. The redirected path could be redirected again as long as the optimal factor $\alpha$ is satisfied.

Overhearing lets all the redirecting nodes, redirectors, know exactly who are redirecting on the same hop as them. Thus, they actually establish a coalition in which these nodes work together to optimize the transmission power. Due to their different locations along the route, the join of each node would make different improvement on the overall sending power. Based on this fact, the payoff for each node would be different. How to allocate the rewards within a coalition for a cooperative game is what the Shapley Value covers.

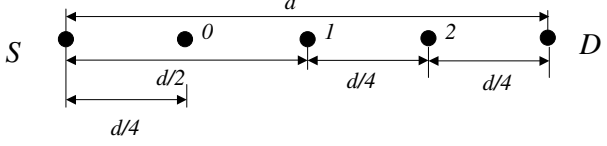

Figure 1: A simple topology of 5 nodes.

\section{B. Calculate the Shapley Value}

Since the path optimization works iteratively, we get one redirector in each iteration. The number of redirectors forming a coalition is related to the node density and the chosen optimal factor. We apply the Shapley Value to allocate the payoff for each member in a redirector coalition. The more power saved because of the participation of a node the more payoff it earns.

For instance, in figure 1, we have a source node $\mathrm{S}$ and a destination node $\mathrm{D}$ with distance $\mathrm{d}$ from $\mathrm{S}$ to $\mathrm{D}$. Three node, $0,1,2$, are lined up from $\mathrm{S}$ to $\mathrm{D}$ with $\frac{1}{4} d$ apart from each other. $P$ is the minimum power that $\mathrm{S}$ uses to reach $\mathrm{D}$. After first redirection, node 1 will be elected as the intermediate relay node. In the following round node 0 would redirect the path from $\mathrm{S}$ to 1 and node 2 would redirect the route from 1 to $\mathrm{D}$. Then we get the coalition formed by node $0,1,2$.

possiblecoalitions $\mathbf{S}=\{0\},\{1\},\{2\},\{0,1\},\{0,2\},\{1,2\},\{0,1,2\}$

Each redirection decreases the total transmission power. We use the total saved power comparing to $P$ as the payoff value of each coalition. The two-ray ground reflection model is used for the calculations. $v(S)$ is the value of a coalition $\mathrm{S}$.

$$
\begin{gathered}
v(0)=v(2)=\frac{174}{256} P \\
v(1)=\frac{224}{256} P \\
v(0,1)=\frac{238}{256} P \\
v(0,2)=\frac{238}{256} P \\
v(1,2)=\frac{238}{256} P \\
v(0,1,2)=\frac{252}{256} P
\end{gathered}
$$

We obtain the contribution of each node by substituting the Shapley Value function. 
$\phi_{0}(v)=\frac{0 ! 2 !}{3 !}\left(\frac{174}{256}-0\right) P+\frac{1 ! 1 !}{3 !}\left(\frac{238}{256}-\frac{224}{256}\right) P+\frac{1 ! 1 !}{3 !}\left(\frac{238}{256}-\frac{174}{256}\right) P+$ $\frac{2 ! 0 !}{3 !}\left(\frac{252}{256}-\frac{238}{256}\right) P=\frac{227}{768} P$

Likewise,

$$
\begin{aligned}
& \phi_{1}(v)=\frac{302}{768} P \\
& \phi_{2}(v)=\frac{227}{768} P
\end{aligned}
$$

From the shapley value of each node, we notice that node 1 is more important than other two nodes since it makes the total transmission power decrease dramatically. So the payoff of node 1 should be more than other members in the grand coalition, which includes every routing node. Another interesting observation is that the grand coalition, $\{0,1,2\}$, can guarantee more value than any other coalitions. This is desirable because it gives a stimulus to each node to cooperate with others.

\subsubsection{Role rotation}

Periodically, every node broadcast hello messages, which include its accumulated wealth so far. A node compares its own wealth with its neighbors'. If it is among the top richest nodes in its neighborhood, it may lose the inspiration to make more money. It can turn off its radio to go to sleep, while other nodes keep working to lift up their ranks.

How many nodes can go to sleep within a neighborhood is decided by a threshold value $\gamma$ of the rich ratio, $R_{\text {rich }}$. We define $R_{\text {rich }}$ as the rank against the number of neighbors so that $\gamma$ is a value in $[0,1]$ (it is also expressed as percentage). A node does not need to get global information in order to make a decision. It checks local information about the wealth of its neighbors and compare its $R_{\text {rich }}$ with $\gamma$. If the $R_{\text {rich }}$ is below $\gamma$, it is rich enough to turn off the radio for a while. The value of $\gamma$ can be chosen while an ad hoc network just starts by using some distributed consensus algorithms. We assume every node already knows $\gamma$ in our simulations. This ratio actually determines the sleeping node portion. Please note that to simplify the protocol design and implementation, we just let nodes decide locally while there are other methods which can be adopted to ensure possible network connectivity requirements.

$$
R_{\text {rich }}=\frac{\text { RankofRichness }}{\text { NumberofNeighbors }}
$$

Let's define the network lifetime, $T_{\text {lifetime }}$, as the time length until the first node runs out of battery. Ideally, it has a relation with $\gamma$ as below, as long as each node has an equal opportunity to sleep.

$$
T_{\text {lifetime }}=\frac{1}{1-\gamma}
$$

We demand that the sleeping nodes wake up before the working nodes check their ranks again after a certain period, which is controlled by a working timer. The just-wake-up nodes broadcast their hello message to advertise their existence and wealth, and listen to hello messages from others. But they do not response to the routing request at this time, because they may still be eligible to sleep soon. The length of this time period is controlled by a listening timer which is set at least one time slot of the hello timer. The total time period of sleeping and listening timers is equal to the working timer so that all nodes review their local richness list and make decision at the same time.

$$
T_{\text {working }}=T_{\text {sleeping }}+T_{\text {listening }}
$$

The downside of CAP-SV is that some routes may be broken due to the going-to-sleep nodes. However in our simulations, we observe the delivery rate just goes down slightly. Considering the extended network lifetime, this is acceptable.

\subsubsection{Dismiss non-working nodes}

We notice that some nodes may always fail to offer a better redirected route due to their positions. Then they do not have a chance to earn any payment. This is also observed in our simulations. It is a waste that they just turn on their radios all the time but do not forward any packets. To cope with this, we let them check whether the connectivity of a network can be taken care of by the working nodes, which outbid them in route redirections. If the connectivity would not be degraded, they can turn off their radios. That gives more nodes an opportunity to save power without causing network partition.

\section{ANALYSIS}

We assume that only when an intermediate node has really forwarded a data packet, is the payment made to it. Also we assume source nodes and destination nodes do not cheat. Under these assumptions, the mechanism of CAP$\mathrm{SV}$ is truthful. To prove this we take a look at two kinds of nodes, normal routing nodes that are chosen in the route discovery phase by RREQs and RREPs, and redirectors that win the competitions with other nodes on each hop.

When a node receives a RREP, it has been picked as a normal router along a route from a source to a destination. It would be paid a certain amount of payment to cover the cost incurred by data transmission. It has two strategies to respond. One is to drop the packet, another is to accept and forward the packet to its predecessor. If a node drops the packet, the route discovery may start again after the source node does not get any replies for a while. A different route going through other nodes would be found. Therefore that node would lose the payment for service providers. Obviously, it is the best interest of this kind of nodes to accept to be a normal routing node and forward the route reply messages correctly.

Normal routing nodes always get payment for forwarding data packets after they are selected along a route. The following route redirection process would have no impact on their payments. So they play truthfully, which means the two end nodes of a hop are truthful nodes. 
When an intermediate node, $N_{i}$, overhears a route reply message being sent from node $N_{h}$ to $N_{g}$, it checks the emission power information and calculates the minimum transmission power it uses to reach the next node, $N_{h}$, on the redirected route. It knows the minimal power used to communicate with the predecessor node, $N_{g}$, because $N_{g}$ must have broadcast a RREQ in the past. Then it may decide what strategies it wants to play. Doing nothing would bring no payment back. Thus, it could either play correctly by sending a route redirection message to $N_{g}, N_{h}$ with true power information, or play incorrectly by sending false power values in the message.

Let us suppose $N_{i}$ tries to cheat with false power information to boost its chance to be a redirector. It may decrease the minimum aggregate power in the redirection message. If this makes $N_{i}$ to be the redirector, it would be out of the communication range of either $N_{g}$ or $N_{h}$ and the data transmission would fail. Then there is no pay to $N_{i}$ due to the payment system assumption. Increasing the minimum power information would not bring any more benefit to $N_{i}$ either because if it is good enough to be a redirector, this may only reduce its payoff for the redirection. Even worse it may be kicked out of the redirector coalition due to the higher total power. If $N_{i}$ cannot offer a better power improvement on this hop comparing to some other node, increasing the minimum aggregate power would only cause it to be outbid definitely.

Therefore, an intermediate node can only maximize its payment by playing truthfully.

\section{PERFORMANCE EVALUATION}

To measure the performance of CAP-SV, we simulated it in different scenarios and analyzed the results using AODV as a reference. We observe that CAP-SV overwhelms AODV on network lifetime though its packet delivery rate is lightly lower.

\subsection{Simulation Environment}

We simulate CAP-SV in ns-2 network simulator [2] with the wireless extension of Monarch project [1]. CAP-SV runs as a routing protocol on the top of IEEE 802.11 MAC layer which uses Channel Sense Multiple Access with Collision Avoidance(CSMA/CA). Before the data transmission, RTS-CTS are exchanged between the sender and receiver pair. In our scenarios, the data packets are transmitted using dynamic minimum power while RTS and CTS are always sent with maximum transmission power.

Every node in our simulations has radio with $2 \mathrm{Mbps}$ bandwidth and 250-meter communication range. We run the CAP-SV in a $500 \mathrm{~m} \times 500 \mathrm{~m}$ area with 20,40 and 60 randomly positioned static nodes. Initially each node has 100 joules battery energy. The transmission cost $1.4 \mathrm{~W}$ power; $1 \mathrm{~W}$ is used for receiving; idle power consumption is $0.83 \mathrm{~W}$ and the power level of a sleeping node is $0.13 \mathrm{~W}$. This energy model is the same as that of SPAN [4]. We also simulated AODV [26] in the same scenarios. All the nodes do not move in our experiments. Besides the routing nodes we have 8 senders and 8 receivers randomly located on different sides of the area in order to have multiple hops between the source and destination nodes. Each sender sends a CBR flow to a

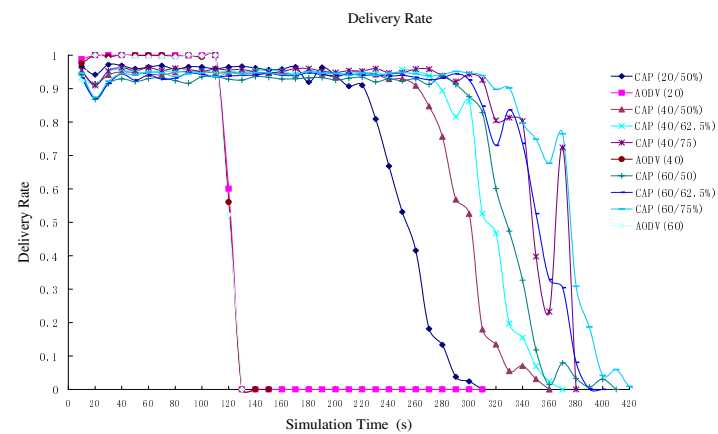

Figure 2: Simulation time vs. packet delivery rate

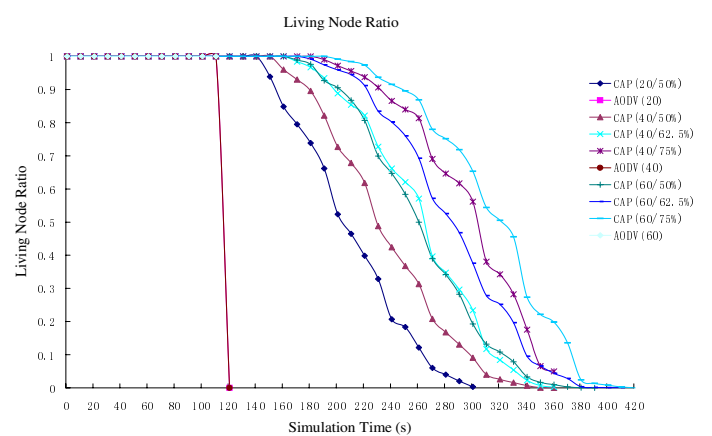

Figure 3: Simulation time vs. living node ratio

paired receiver on the other side, and each CBR flow sends three 128-byte packets per second. These 16 senders and receivers have enough energy to assure they would not run of power before any of the routing nodes. We set the sleeping period to be 8.8 seconds and after being woken up, a node listens for 1.2 seconds. The active nodes are working for 10 seconds before checking their neighbor list. By setting the timers in this way, all the nodes will make decisions on whether or not shut down their radios at the same time.

We have simulated 20, 40 and 60 routing nodes with CAPSV and AODV respectively. In 40 and 60 routing node scenarios we vary the rich ratio threshold $\gamma$ to be $50 \%, 62.5 \%$ and $75 \%$ for CAP-SV. In the figures, CAP-SV(40/50\%) depicts that the scenario has 40 routing nodes with $50 \%$ rich ratio using CAP-SV. Likewise, AODV(40) denotes the 40node experiment with AODV. In the 20-node scenario, we just remain $\gamma$ as $50 \%$ in order to avoid early network partitions.

We measure the simulation results by three metrics, delivery rate, living node ratio and left energy per living node, which are drawn in figure 2, 3, and 4 . Each point in the figures is the average of 30 runs.

\subsection{Result Analysis}

Packet delivery rate is the percentage of sent packets received by the intended destinations. In figure 2 , we can see CAP-SV has slightly lower delivery rate than AODV in the first 120 seconds. This is because after every working period all the CAP-SV nodes check whether they are in the 


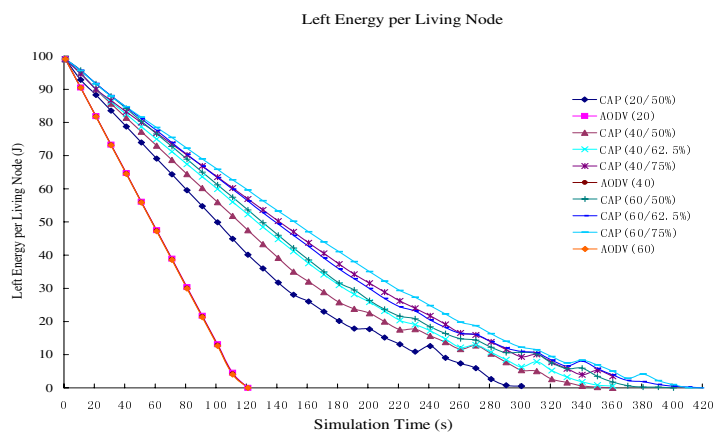

Figure 4: Simulation time vs. left energy per living node

top richest node list. If a node is forwarding packet before it is rich enough to take a nap, an error message is created and sent to its upstream node. This may cause packet dropping in routing nodes along this route. We just allow nodes to drop packets in this case to simplify the protocol, though some methods, such as local repair and retransmission, may be used to prevent the lost packets.

In both AODV and CAP-SV scenarios, the delivery rate $R_{\text {delivery }}$ almost always stays high at first. When it begins to drop, it falls quickly. Since the graphs used in our simulations are dense, the link redundancy is high. When some routing nodes are dead, the packets can be forwarded by a small set of routers. We can see at the same rich ratio threshold $\gamma$, the node density matters. Higher density brings longer network lifetime.

When the $R_{\text {delivery }}$ begins to drop, it does not go down monotonically. The vibration is because that every node decides whether to go to sleep independently without consideration of its position. Then the sleeping nodes may concentrate on the same side of the network and leave the active nodes on the other side. In this case, some senders may not have a router within their radio range. This only happens when few routing nodes are left.

Figure 3 shows that almost all the nodes with AODV burn out their energy at the same time. Since energy efficiency is not an issue in AODV, every node running AODV must be on all the time so that everyone is off together.

In the 20-node scenario, CAP-SV has about $25 \%$ more network lifetime than AODV. This lifetime extension is achieved with the consideration of the incentive of each node. As a network shuts down nodes more aggressively with larger $\gamma$, longer lifetime can be expected. CAP-SV(60/75\%) almost extends the lifetime of a network by $75 \%$. However, increasing $\gamma$ also increases the risk of early network partitions. Another issue that needs to be noted is that CAP-SV needs network flows to trigger the competitions between nodes.

It is perceived in figure 4 that the larger the value of $\gamma$, the flatter the curve of average left energy per living node in CAP-SV. Larger $\gamma$ brings more sleeping nodes, thus the whole energy consumption of a network slows down.

\section{CONCLUSION AND FUTURE WORKS}

We present a scheme to determine the payoff of each node in a cooperative coalition in wireless ad hoc networks using the Shapley Value, which is well known in game theory for allocating cost and payoff for the participants in a cooperative game. By doing so, we show the concept of Shapley value can fit MANET research area very well. Rewarding the contribution with payoff is the motivation of this paper. We believe if the participation of a node as a service provider brings back decent payoff, it is reasonable to expect this node to stay as a supporter for network-wide services though it may intend to be selfish.

We prove the mechanism of deciding payments for routing nodes is truthful. Therefore, only when a node acts correctly by revealing true information, can its interests be best served in the routing protocol CAP-SV.

The simulation results demonstrate that CAP-SV outperforms AODV on network lifetime though the packet delivery rate drops slightly. Our protocol allows each node to make decision locally, which reduces signaling overhead and routing complexity.

Our scheme only addresses how to determine payoff of each node. How to redeem the earning from cooperation and how this affects the network behavior are wealthy of future research works. We believe that game theoretical concepts give us novel approaches to model and analyze wireless ad hoc networks with selfish nodes.

\section{REFERENCES}

[1]CMU Monarch Extensions to ns. http://www.monarch.cs.cmu.edu/

[2]ns Notes and Documentations.

http://www.isi.edu/vint/nsnam/

[3]R. Wattenhofer, L. Li, V. Bahl and Y.M. Wang. Distributed Topology Control for Power Efficient Operation in Multihop Wireless Ad Hoc Networks. in Proc. of IEEE INFOCOM, pp. 1388-1397, April 2001.

[4]Benjie Chen, Kyle Jamieson, Hari Balakrishnan, and Robert Morris. Span: An energy-efficient coordination algorithm for topology maintenance in ad hoc wireless networks. in Proc. of the 7th ACM Int'l Conf. on Mobile Computing and Networking, pp. 85-96. Rome, Italy, July 2001.

[5]J. Gomez, A.T. Campbell, M. Naghshineh and C. Bisdikian. Conserving Transmission Power in Wireless Ad Hoc Networks. In Proc. 9th International Conference on Network Protocols (ICNP 2001), Riverside, California, November 11 - 14, 2001.

[6]Eun-Sun Jung and Nitin H. Vaidya. A Power Control MAC Protocol for Ad Hoc Networks. in Proceedings of the eighth annual international conference on Mobile computing and networking (MOBICOM), pp.36-47, Atlanta, U.S.A., September 2002.

[7]Y. Xu, J. Heidemann, and D. Estrin. Geography-informed energy conservation for ad hoc routing. In Proceedings of the ACM/IEEE International Conference on Mobile Computing and Networking, pp. 70-84, Rome, Italy, July 2001. [8]Suresh Singh, Mike Woo, and C. S. Raghavendra. PowerAware Routing in Mobile Ad Hoc Networks. In Proc. of The Fourth Annual ACM/IEEE International Conference on Mobile Computing and Networking, pp. 181-190, Dallas, 
TX, October 1998.

[9]Levente Buttyan, Jean-Pierre Hubaux. Nuglets:a Virtual Currency to Stimulate Cooperation in Self-Organized Mobile Ad Hoc Networks. technical report DSC/2001/001, Swiss Federal Institute of Technology, Lausanne, Department of Communication Systems, Jan 2001.

[10]N. Ben Salem, L. Buttyan, J.-P. Hubaux, and M. Jakobsson. A charging and rewarding scheme for packet forwarding in multi-hop cellular networks, in Proc. of the 4th ACM/SIGMOBILE MobiHoc, pp. 13 - 24. Annapolis, Maryland, June, 2003.

[11]Alberto Cerpa and Deborah Estrin," ASCENT: Adaptive Self-Configuring sEnsor Networks Topologies,", in Proc. of Infocom 2002, New York, NY USA, June 2002.

[12] Volkan Rodoplu and Teresa H. Meng. Minimum energy mobile wireless networks. in Proceedings of the 1998 IEEE International Conference on Communications, ICC'98, Atlanta, GA, June 1998, vol. 3, pp. 1633-1639.

[13]Sergio Marti, T. J. Giuli, Kevin Lai, and Mary Baker. Mitigating routing misbehavior in mobile Ad hoc networks. In Proceedings of the Sixth annual ACM/IEEE International Conference on Mobile Computing and Networking (MobiCom), pp. 255-265, Boston, Massachusetts, August 2000.

[14]Allen B. MacKenzie, Stephen B. Wicker. Game theory and the design of self-configuring, adaptive wireless networks. IEEE Communications Magazine, no. 11, November 2001 pp. $126-131$

[15]]L.C. Thomas. Games, theory and applications. 1984, Ellis Horwood Limited, England.

[16]Sergiu Hart, Advance in Value Theory, in Game Theory and Applications, pp. 166-175, 1990, Academic press, Inc. [17]Avinash Dixit, Susan Skeath, Games of Stratey, 1999, W.W.Norton \& Company, Inc.

[18]R. Kravets, P. Krishnan. Application-driven Power Management for Mobile Communication. In Proc. of the Fourth Annual ACM/IEEE International Conference on Mobile Computing and Networking (MobiCom), Dallas, TX, Oct. 1998, pp. 263-277.

[19]John G Dorsey, Game-theoretic resource allocation in mobile ad hoc networks, Doctoral thesis prospectus, department of electrical and computer engineering, Carnegie Mellon University, Pittsburgh, PA, Dec 2001.

[20] Errol L. Lloyd, Rui Liu, Madhav V. Marathe, Ram Ramanathan, S. S. Ravi, Algorithmic aspects of topology control problems for ad hoc networks. In Proc. of the third ACM international symposium on Mobile ad hoc networking \& computing, pp. 123 - 134, Lausanne, Switzerland, June 2002.

[21]Joan Feigenbaum, Scott Shenker, Distributed Algorithmic Mechanism Design: Recent Results and Future Directions. Invited talk in DIAL-M'02, Sep. 28, 2002, Atlanta, Georgia.

[22] Laura Marie Feeney, Martin Nilsson, Investigating the energy consumption of a wireless network interface in an ad hoc networking environment. IEEE INFOCOM 2001, no. 1, April 2001 pp. 1548-1557.

[23]Christine E. Jones, Krishna M. Sivalingam, Prathima Agrawal, Jyh Cheng Chen. A survey of Energy Efficient Network Protocol for Wireless Network. Wireless Networks, 7(4), pp.343-358, July 2001.

[24]Seung Yi, Prasad Naldurg, Robin Kravets, Security-Aware Ad Hoc Routing For Wireless Networks, Technical Report

UIUCDCS-R-2001-2241, Department of Computer Science,
UIUC, August 2001

[25]Panagiotis Papadimitratos, Zygmunt J. Hass, Secure Routing for Mobile Ad Hoc Networks. in Proc. of SCS Communication Networks and Distributed Systems Modeling and Simulation Conference (CNDS 2002), San Antonio, TX, January 2002.

[26] S. Das, C.E. Perkins and E. M. Royer. Ad hoc On Demand Distance Vector(AODV) Routing. Mobile Ad-hoc Network (MANET) Working Group, IETF, January 2002. [27]D. Johnson, D. Maltz, Y.-C. Hu, and J. Jetcheva. The dynamic source routing protocol for mobile ad hoc networks. IEEE Internet Draft, March 2001. draft-ietf-manet-dsr-05.txt. [28]Theodore S. Rappaport, Wireless Communications: Principles and Practice, Prentice Hall, 1996.

[29]L. Anderegg, S. Eidenbenz, Ad hoc-VCG: a truthful and cost-efficient routing protocol for mobile ad hoc networks with selfish agents, in Proceedings of the 9th annual international conference on Mobile computing and networking, pp.245 - 259, San Diego, CA, USA

[30]N. Nisan, A. Ronen, Algorithmic mechanism design, in Games and Economic Behavior 35, pp.166-196, 2001.

[31]C. Ambuehl and A. Clementi and P. Penna and G. Rossi and R. Silvestri, Energy Consumption in Radio Networks: Selfish Agents and Rewarding Mechanisms, in Proc. of The 10th Colloquium on Structural Information and Communication Complexity (SIROCCO 2003), Umea, Sweden, June 2003.

[32]K. Jain and V. Vazirani, Applications of approximation algorithms to cooperative games. In Proc. of $33 \mathrm{rd} \mathrm{ACM}$ Symposium on Theory of Computing(STOC01), pp.364-372, Crete, Greece, July 2001.

[33]J. Feigenbaum, C. Papadimitriou, and S. Shenker. Sharing the cost of multicast transmissions. In Journal of Computer and System Sciences 63(1), pp.21-41, 2001. 